OPEN ACCESS

Edited by:

Juan Bautista De Sanctis,

Palacký University Olomouc, Czechia

Reviewed by:

Maria Fatima Garcés,

Central University of Venezuela,

Venezuela

Diego Franco,

University of Jaén, Spain

William Bracamonte,

Texas Tech University Health

Sciences Center, United States

${ }^{*}$ Correspondence: Aisong Zhu

liaoningzhongyi@hotmail.com Wenna Chen

chenwn1992@126.com;

chenwn@/nutcm.edu.cn

Specialty section:

This article was submitted to Cytokines and Soluble Mediators in Immunity,

a section of the journal

Frontiers in Immunology

Received: 04 June 2020

Accepted: 10 August 2020

Published: 10 September 2020

Citation:

Li X, He X, Wang J, Wang $D$, Cong P, Zhu A and Chen W (2020) The Regulation of Exosome-Derived miRNA on Heterogeneity of Macrophages in Atherosclerotic Plaques. Front. Immunol. 11:2175. doi: 10.3389/fimmu.2020.02175

\section{The Regulation of Exosome-Derived miRNA on Heterogeneity of Macrophages in Atherosclerotic Plaques}

\author{
Ximing $\mathrm{Li}^{1}$, Xinyong $\mathrm{He}^{1}$, Junyan Wang ${ }^{2}$, Dan Wang ${ }^{3}$, Peiwei Cong ${ }^{1}$, Aisong $\mathrm{Zhu}^{4 *}$ and \\ Wenna Chen ${ }^{1,3 *}$ \\ ${ }^{1}$ Key Laboratory of Ministry of Education for TCM Viscera-State Theory and Applications, Liaoning University of Traditional \\ Chinese Medicine, Shenyang, China, ${ }^{2}$ The First Medical College, Guangzhou University of Chinese Medicine, Guangzhou, \\ China, ${ }^{3}$ Department of Medical Science of Laboratory, Liaoning University of Traditional Chinese Medicine, Shenyang, \\ China, ${ }^{4}$ Basic Medical College, Zhejiang Chinese Medical University, Hangzhou, China
}

Exosomes are nanosized vesicles secreted by most cells, which can deliver a variety of functional lipids, proteins, and RNAs into the target cells to participate in complex intercellular communications. Cells respond to certain physical, chemical, and biological stimuli by releasing exosomes. Exosomes are rich in small molecules of RNA, including miRNAs and mRNAs, which have been demonstrated to have certain functions in recipient cells. Recent studies on single-cell RNA sequences have revealed the transcription and the heterogeneity of macrophages in Ldlr-/-mice fed with a highfat diet. Five macrophage populations were found in the atherosclerotic plaques. It is worth noting that these subset populations of macrophages seem to be endowed with different functions in lipid metabolism and catabolism. A total of 100 differentially expressed mRNAs were selected for these subset populations. Importantly, these macrophage populations were also present in human advanced atherosclerosis. To clarify the specific functions and the regulatory mechanism of these macrophage populations, we extracted exosome RNAs from the plasma of patients with chronic coronary artery disease (CAD) and performed RNA sequencing analysis. Compared with the healthy control, a total of 14 miRNAs were significantly expressed in these patients. A total of 5,248 potential mRNAs were predicted by the bioinformatics platform. Next, we determined the outcome of the intersection of these predicted mRNAs with 100 mRNAs expressed in the above-mentioned five macrophage populations. Based on the screening of miRNA-mRNA pairs, a co-expression network was drawn to find out the key RNAs. Three down-regulated miRNAs and five up-regulated mRNAs were selected for validation by real-time RT-PCR. The results showed that the expression of miR-4498 in plasma exosomes was lower than that in the healthy control, and the expressions of Ctss, Ccr2 and Trem2 mRNA in peripheral blood mononuclear cells isolated from CAD patients were higher. In order to clarify the regulatory mechanism, we established 
a co-culture system in vitro. Studies have shown that the uptake of exosomes from CAD patients can up-regulate the expression of Ctss, Trem2, and Ccr2 mRNA in THP-1 cells induced by lipopolysaccharide. Our findings revealed a unique relationship between the transcriptional signature and the phenotypic heterogeneity of macrophage in the atherosclerotic microenvironment.

Keywords: exosomes, macrophages, miRNA, atherosclerosis, networks

\section{INTRODUCTION}

Exosomes are nanosized vesicles secreted by most cells, which can deliver a variety of functional lipids, proteins, and RNAs into the target cells to participate in complex intercellular communications. Cells respond to a series of physical, chemical, and biological stimuli (such as inflammation, oxidative stress, and hypoxia) by releasing exosomes. Exosomes are rich in small molecules of RNA, including miRNAs and mRNAs, which have been demonstrated to have certain functions in recipient cells. The exosomes of RNAs can change the gene expression in those cells (1).

Many studies have shown that the release of exosomes following ischemic injuries affects not only the cardiovascular cells but also the cells in the microenvironment, thereby modulating the repair process (2). Exosomes could regulate the differentiation, proliferation, and remodeling of cardiomyocytes, fibroblasts, and inflammatory cells (3-5). As a kind of lipid mediators, exosomes can deliver lipids and lipolytic enzymes, and their biosynthesis requires specific lipids and membrane reorganization. This study aimed to provide a comprehensive insight into chronic inflammatory diseases to further understand the importance of exosomes in solving inflammatory response and to explore new regulatory mechanisms.

It was found that several functional phenotypes of macrophages respond to the microenvironment and play different roles in vascular inflammation and atherosclerosis. Various macrophage populations were found in atherosclerotic plaques (6). It is worth noting that these macrophage subset populations seemed to be endowed with different functions in lipid metabolism and catabolism.

To clarify the specific functions and the regulatory mechanism of these macrophage subsets and the regulatory mechanism, we set out to reveal the unique relationship between the transcriptional signature and the phenotypic heterogeneity of macrophages in the atherosclerotic microenvironment.

\footnotetext{
Abbreviations: ApoE, apolipoprotein E; AS, atherosclerosis; CAD, coronary artery disease; CCL, C-C motif chemokine ligand; CXCL2, C-X-C motif chemokine ligand 2; FACS, fluorescence-activated cell sorting; GO, Gene Ontology; IL, interleukin; KEGG, Kyoto Encyclopedia of Genes and Genomes; LDLR, lowdensity lipoprotein receptor; LPS, lipopolysaccharide; miRNA, microRNA; MoDC, monocyte-derived dendritic cells; MRE, microRNA response element; $\mathrm{M} \varphi$, macrophages; NLRP3, nucleotide-binding oligomerization domain, leucine-rich repeat and pyrin domain containing 3; NTA, nanoparticle tracking analysis; PMA, phorbol-12-myristate-13-acetate; RNA-seq, RNA sequencing; RT-PCR, reverse transcription-polymerase chain reaction; TLR2, toll-like receptor 2; TREM2, triggering receptor expressed on myeloid cells.
}

\section{MATERIALS AND METHODS}

\section{Sample Collection}

Cases of patients of stable angina (SA) with significant coronary artery stenosis $(>50 \%)$ were collected $(n=15)$. The age of the patients ranged from 45 to 75 years old. No patient was under medication with corticosteroids or non-steroidal anti-inflammatory drugs, except for aspirin. No patient had concurrent inflammatory or neoplastic conditions likely to be associated with an acute-phase response. Selective coronary angiography was performed in multiple views using the standard Judkins techniques to figure out the number of coronary artery stenosis in terms of single- or multiple-vessel stenosis. The ethical approval for the study was authorized by the institutional ethical committee of Liaoning University of Traditional Chinese Medicine, and all participants provided informed consent for the experiment. The control group in this study was composed of age-matched healthy volunteers $(n=15)$ compared to patients with stable angina. The demographic and clinical data are shown in Table 1.

\section{RNA Isolation and Sequencing}

Total Exo-RNAs were extracted from the plasma derived from four individuals in the coronary artery disease (CAD) group or the control group. ExoRNeasy serum/plasma Maxi kits (QIAGEN, Cat. No. 77064) were used according to the instruction. Pre-filtered (with $0.8-\mu \mathrm{m}$ filter) plasma was used to exclude cell contamination and mixed with the same volume of $\mathrm{XBP}$ buffer. The exosomes were bound to an exoEasy membrane affinity spin column after centrifuging at $500 \times g$ for $1 \mathrm{~min}$. Then, the bound exosomes were washed with $10 \mathrm{ml}$ XWP buffer by centrifuging at $500 \times g$ for $1 \mathrm{~min}$. A total of $700 \mu \mathrm{l}$ QIAzol was added to the membrane. Spinning went on for $5 \mathrm{~min}$ at $5,000 \times g$ to collect the lysate, which was transferred completely to a 2 -ml tube. Then, $90 \mu \mathrm{l}$ chloroform was added to the tube containing the lysate for $15 \mathrm{~s}$, followed with centrifuging for $15 \mathrm{~min}$ at $12,000 \times g$ at $4^{\circ} \mathrm{C}$. The upper aqueous phase was transferred to a new collection tube, and two volumes of $100 \%$ ethanol were added prior to mixing thoroughly. Up to $700 \mu \mathrm{l}$ of the sample was pipetted into a RNeasy MinElute spin column in a 2 -ml collection tube and centrifuged at $\geq 8,000 \times g$ for $15 \mathrm{~s}$ at room temperature (RT). Then, the flow-through was discarded. RWT buffer at $700 \mu \mathrm{l}$ was added to the column and centrifuged for $15 \mathrm{~s}$ at $\geq 8,000 \times g$. A total of $500 \mu \mathrm{l}$ of RPE buffer was pipetted onto the column and centrifuged for $15 \mathrm{~s}$ at $\geq 8,000 \times g$. The step was repeated and centrifugation was carried out for $2 \mathrm{~min}$ at $\geq 8,000 \times g$. Then, $14 \mu \mathrm{l}$ of 
TABLE 1 | Demographic and clinical characteristics of the coronary artery disease (CAD) patients and the controls.

\begin{tabular}{lcc}
\hline Variable & Control $(\boldsymbol{n}=\mathbf{1 5})$ & CAD $(\boldsymbol{n}=\mathbf{1 5})$ \\
\hline Age (year) & $56 \pm 11$ & $55 \pm 10$ \\
Sex (M/F) & $10 / 5$ & $9 / 6$ \\
Smokers, $n$ (\%) & $5(33)$ & $4(26)$ \\
Hypertension, $n$ (\%) & $8(53)$ & $3(20)$ \\
Diabetes mellitus, $n$ (\%) & $3(20)$ & 0 \\
Coronary artery stenosis (\%) & $61 \pm 13$ & $8 \pm 5^{\star *}$ \\
TC (mmol/L) & $4.43 \pm 0.72$ & $5.05 \pm 0.94$ \\
TG (mmol/L) & $0.94 \pm 0.41$ & $1.96 \pm 1.04^{*}$ \\
LDL-C (mmol/L) & $2.84 \pm 0.79$ & $3.43 \pm 0.66^{*}$ \\
HDL-C (mmol/L) & $1.44 \pm 0.37$ & $1.18 \pm 0.32$ \\
Apo A1 (mmol/L) & $1.27 \pm 0.33$ & $0.95 \pm 0.22$ \\
Apo B (mmol/L) & $0.92 \pm 0.32$ & $0.87 \pm 0.23$ \\
FBG (g/L) & $2.91 \pm 0.85$ & $2.16 \pm 0.73$
\end{tabular}

TC, total cholesterol; TG, triglyceride; LDL-C, low-density lipoprotein cholesterol; $H D L-C$, high-density lipoprotein cholesterol; FBG, fibrinogen; Apo A1, apolipoprotein A1; Apo B, apolipoprotein $B$. ${ }^{* *} p<0.01$ or ${ }^{*} p<0.05$ vs. control.

RNase-free water was added directly to the column membrane, and the mixture was centrifuged for $1 \mathrm{~min}$ to elute the RNA. The RNA concentration was detected by the RNA Assay Kit (Life Technologies, CA, United States).

miRNA-seq was analyzed by Genesky Biotechnologies Inc., Shanghai. The libraries for RNA-seq were performed according to the Illumina Truseq small RNA protocol and sequenced with HiSeq 3000 (Illumina Inc.). Mirdeep2 software ${ }^{1}$ (7) was used to compare the small RNA sequence of each sample with the miRNA precursor and the mature sequence of the corresponding species in the miRBase database ${ }^{2}$. The known miRNAs and their secondary structure were identified by comparison with the homologous miRNA sequences of the related species. The counting numbers of the known miRNAs in each sample were calculated. The target genes of the miRNAs were predicted by TargetScan ${ }^{3}$ and StarBase ${ }^{4}$.

\section{Differential Analysis on the Expression of mRNAs in Monocyte-Related Populations in AS Plaques}

Clément performed single-cell RNA sequencing analysis on $\mathrm{CD} 45^{+}$cells extracted from the aorta of chow-fed mice and atherosclerotic $\mathrm{Ldll}^{-/-}$mice fed with a high-fat diet for 11 weeks $(n=10)(6)$. The aortas were pooled to generate the samples used for single-cell RNA sequencing. Then, a two-dimensional space through $t$-stochastic neighbor embedding (t-SNE) was used to identify overlapping and atherosclerosis-associated immune cell populations. Thirteen distinct aortic cell clusters were singled out. In the myeloid cell populations, five monocyterelated populations were found, including resident macrophages, monocytes, monocyte-derived dendritic cells, inflammatory

\footnotetext{
${ }^{1}$ https://www.mdc-berlin.de/8551903/en/

${ }^{2}$ http://www.mirbase.org/

${ }^{3} \mathrm{http}: / /$ www.targetscan.org

${ }^{4}$ http://starbase.sysu.edu.cn
}

macrophages, and triggered receptor expressed on myeloid cells 2 (TREM ${ }^{\text {hi }}$ ) macrophages. Differential gene expression and gene ontology enrichment were analyzed to distinguish from these five clusters. A heatmap plot was drawn using BioJupies, a website that automatically generates RNA-seq data analysis (8), to show the differentially expressed genes characteristic of the five clusters of monocyte-related populations. In the clustering analysis, the up-regulated and the down-regulated genes were colored in red and blue, respectively. To find out the key mRNAs, we generated the diagram with Venny 2.1.0 to show the number of unique and shared mRNAs experimentally identified in the five clusters.

\section{GO and KEGG Analysis of DEmRNAs in Aortas of Mice}

We used the Gene Ontology (GO) database ${ }^{6}$ to perform GO analysis on the mRNAs. The $P$-value and the false discovery rate were calculated by Fisher's exact test and multiple-comparison test, respectively $(p<0.05)$. Around 1,342 differentially expressed genes were classified in the light of the GO terms, including biological process, molecular function, and cellular component.

Pathway enrichment analyses in the aortas of mice were performed based on Kyoto Encyclopedia of Genes and Genomes (KEGG) pathway analysis by using either chi-square test or Fisher's exact test. The pathways with more annotations in the differentially expressed genes $(p<0.05)$ were considered to be significantly enriched and used for the biological identification of each cluster (scale: $\log 2$ fold change).

\section{MiRNA-Seq Analysis of Plasma Exosomes From CAD Patients and Healthy Control}

MiRNA-seq was analyzed, and a million counts of mapped reads for each sample were noted. The Fastq reads were checked for quality, and an interquartile range (IQR) plot, a volcano plot, and a scatter plot were drawn using BioJupies, a website which automatically generates RNA-seq data analysis (8). A principal component analysis (PCA) revealed the overall distribution of differentially expressed miRNAs. The differentially expressed miRNAs in each sample were shown in the heatmap plot.

\section{Constructing a miRNAs-mRNAs Interacted Network}

We identified the intersection of common mRNAs among DEmRNAs and the predicted mRNAs by miRNAs. Co-expression networks were drawn by using Cytoscape 3.7.1 software based on the screening of miRNA-mRNA pairs.

Since miRNA response elements (MREs) are the mediators of mRNA-miRNA interaction, we utilized miRWalk 2.0 to identify the relationships between the 14 DEmiRNAs and the 1,342 DEmRNAs (9). Furthermore, we selected the pairs with strong correlations to construct the miRNAs-mRNAs network. The interacted networks indicated the co-expression patterns of miRNAs and mRNAs. In this network, each gene corresponds

\footnotetext{
${ }^{5}$ https://bioinfogp.cnb.csic.es/tools/venny/index.html

${ }^{6} \mathrm{http}: / /$ www.geneontology.org
} 
to a node, and the connection of two genes is represented by an edge which indicates a significantly negative correlation. The main miRNAs and mRNAs in the network are shown in different colors.

The highchart was set out ${ }^{7}$ to visualize the relationships among miRNAs, mRNA, and five monocyte-related populations. Each point consists of multiple-weighted links to other points in this chart. The key nodes were selected by dependency wheel series $(\geq 5)$ in this network.

\section{Isolating Exosomes From Plasma}

Exosomes were isolated from equal amounts ( 2 to $3 \mathrm{ml}$ ) of plasma (containing an anticoagulant, ethylenediaminetetraacetic acid) from control or CAD patients ( $n=3$ /group) using exoEasy ${ }^{\circledR}$ Serum/Plasma Maxi Kit (Cat. No. 76064, QIAGEN GmbH, Hilden, Germany). The individuals were consistent with the ones for exo-RNA sequencing. Pre-filtered (with $0.8-\mu \mathrm{m}$ filter) plasma was used to exclude cell contamination and mixed with the same volume of XBP buffer. The exosomes were bound to an exoEasy membrane affinity spin column after centrifuging at $500 \times g$ for $1 \mathrm{~min}$. Then, the bound exosomes were washed with $10 \mathrm{ml}$ XWP buffer by centrifuging at $500 \times g$ for $1 \mathrm{~min}$. Then, the exosomes were eluted with $400 \mu \mathrm{l} \mathrm{XE}$ buffer and were then ready for physical analysis or uptake by the recipient cells. The isolated exosomes were characterized by transmission electron microscopy, nanoparticle tracking analysis (NTA), and Western blot.

\section{Observation of Exosomes With Transmission Electron Microscopy}

The exosomes re-suspended in phosphate-buffered saline (PBS) were loaded onto Formvar-carbon-coated electron microscopy grids that had been glow-discharged for $30 \mathrm{~s}$ in air. Then, the grids were immediately negatively stained using $2 \%$ phosphotungstic acid and visualized with an H-7650 transmission electron microscope (HITACHI, Tokyo, Japan) operated at $80 \mathrm{kV}$.

\section{Nanoparticle Tracking Analysis of Exosomes}

The size distribution of exosomes was assayed using NanoSight N300 (Malvern Instruments, Malvern, United Kingdom). The samples were monitored with the use of a $640-\mathrm{nm}$ laser. The frame rate used was 30 frames per second and Nanosight particle tracking software (version NTA 3.2) was used to calculate exosome concentrations and size distribution.

\section{Analysis of Exosome Markers by Western Blot}

Protein was extracted from isolated exosomes with RIPA buffer (Sigma-Aldrich), and a total of $20 \mu \mathrm{g}$ of protein was loaded into $10 \%$ SDS-PAGE gel for separation. Then, the protein was transferred onto a polyvinylidene fluoride membrane (Bio-Rad, CA, United States). The membrane was incubated with antiCD9 rRabbit mAb (1:1,000, clone D8O1A, CST, \#13174) or

${ }^{7}$ https://jshare.com.cn/demos/JEtvoW
CD63 (1:1,000, Invitrogen, \#10628D) overnight at $4^{\circ} \mathrm{C}$ after having been blocked with $5 \%$ milk. The secondary antibody was horseradish peroxidase-linked anti-rabbit IgG (1:2,000, CST, \#7074) or anti-mouse IgG (1:2,000, CST, \#7076). The membrane was visualized with ECL Western Blotting Detection (Tanon 5200, Shanghai, China).

\section{Isolating Human Peripheral Blood Mononuclear Cells}

Human peripheral blood mononuclear cells (PBMCs) were isolated in Lymphoprep ${ }^{\text {TM }}$ and SepMate ${ }^{\text {TM }}$ RUO tubes (STEMCELL Technologies, United States) by using density gradient centrifugation. Then, $2.5 \mathrm{ml}$ of blood was diluted with an equal amount of PBS with $2 \%$ fetal bovine serum. The blood was layered on top of $5 \mathrm{ml}$ Lymphoprep ${ }^{\mathrm{TM}}$, being careful to minimize the mixing of blood with Lymphoprep ${ }^{\mathrm{TM}}$. The tubes were centrifuged at $800 \times g$ for $20 \mathrm{~min}$ at room temperature with brake-off. The upper plasma layer was removed and discarded without disturbing the plasma-Lymphoprep ${ }^{\mathrm{TM}}$ interface. The mononuclear cells (MNC) layer was removed and retained at the interface. The MNCs were washed once with RPMI1640 medium. The monocytes were separated from other leukocytes by adherence to plastic after being cultured in the plate for $2 \mathrm{~h}$. The monocytes were collected for mRNA detection by RT-PCR ( $n=3$ /group).

\section{Differentiating THP1 Cells Into Macrophages}

The THP1 cells were differentiated into macrophages cultured in medium 1640 and supplemented with $25 \mathrm{nM}$ phorbol-12myristate-13-acetate (PMA) and 10\% fetal calf serum over $48 \mathrm{~h}$, followed by a recovery period of $24 \mathrm{~h}$ in culture in the absence of PMA (10). The THP1 cells were observed to exhibit macrophagelike characteristics, such as adherent, round, short spindle, or irregular polygons with localized protrusions.

\section{Uptake of Exosome-Derived miRNA by Macrophages}

To examine the uptake of exosomes by THP1 cells in vitro, the exosomes were labeled with a PKH67 Green Fluorescent Cell Linker Mini Kit (Cat. No. MINI67, Sigma-Aldrich). Then, $1 \mathrm{ml}$ of final staining volume contained final concentrations of $1 \times 10^{-6} \mathrm{M}$ of PKH67 and $5 \mu \mathrm{g} / \mathrm{ml}$ exosomes. Moreover, $0.5 \mu \mathrm{g}$ fluorescently labeled exosomes $(5 \mu \mathrm{g} / \mathrm{ml})$ were then added into $1 \times 10^{6}$ macrophages at $37^{\circ} \mathrm{C}$ and incubated for $12 \mathrm{~h}$. Then, the labeled exosomes were captured by living cell imaging. Fluorescent cellular imaging was then performed with Cytation $^{\mathrm{TM}} 1$ Cell Imaging Multi-Mode Reader (BioTek, United States) by using the GFP channel for PKH67 green-fluorescence-labeled exosomes. Cell number counting was detected in the bright field.

\section{RNA Isolation From Cells and Exosomes}

To validate the similarities in miRNA profiles between the exosomes isolated for in vitro experiments and the exo-RNA 
extracted from plasma, we extracted RNA from exosomes isolated from plasma for quantitation of miRNAs by real-time qPCR.

Total RNA was isolated, using miRNeasy Mini Kit (cat\# 217004, Qiagen GmbH, Hilden, Germany), from exosomes or cells. Briefly, the samples were added with $700 \mu \mathrm{l}$ QIAzol lysis reagent and incubated at $25^{\circ} \mathrm{C}$ for $5 \mathrm{~min}$. Chloroform $(140 \mu \mathrm{l})$ was added, and the tubes were shaken vigorously for $15 \mathrm{~s}$. The samples were centrifuged for $15 \mathrm{~min}$ at $12,000 \times g$ at $4^{\circ} \mathrm{C}$ after incubation for 2 to $3 \mathrm{~min}$. The upper aqueous phase was transferred to a new collection tube and added with 1.5 volumes of $100 \%$ ethanol with thorough mixing by pipetting. Up to $700 \mu \mathrm{l}$ of the sample was pipetted into a RNeasy ${ }^{\circledR}$ Mini column in a $2-\mathrm{ml}$ collection tube and centrifuged at $\geq 8,000 \times g$ for $15 \mathrm{~s}$ at RT, and then the flow-through was discarded. Moreover, $700 \mu l$ of RWT buffer was added to the RNeasy Mini column and centrifuged for $15 \mathrm{~s}$ at $\geq 8,000 \times g$, and then the flow-through was discarded. RPE buffer $(500 \mu \mathrm{l})$ was pipetted onto the RNeasy Mini column and centrifuged for $15 \mathrm{~s}$ at $\geq 8,000 \times \mathrm{g}$, and then the flow-through was discarded (repeated this step). The RNeasy Mini column was transferred to a new 1.5-ml collection tube, and 30-50 $\mu$ l RNasefree water was pipetted directly onto the column membrane and then centrifuged for $1 \mathrm{~min}$ at $\geq 8,000 \times g$ to elute.

\section{Validation of DEmiRNAs in Exosomes or DEmRNAs in PBMCs by Real-Time RT-qPCR}

Total RNA was reverse-transcribed to cDNA with AMV reverse transcriptase (Takara) and a stem-loop RT primer. Real-time PCR was performed with a TaqMan PCR kit and ABI 7900 (Applied Biosystems). Exo-miRNA quantification was conducted by using the miScript II RT Kit (QIAGEN) and the miScript SYBR ${ }^{\circledR}$ Green PCR Kit (QIAGEN). The RNA levels were determined by $2^{-\Delta \Delta C t}$ method and normalized to $\beta$-actin and U6 for mRNAs and miRNAs, respectively ( $n=3$ /group). The mature sequences of miR-4498, miR-1226-5p, and miR-320c were UGGGCUGG CAGGGCAAGUGCUG, GUGAGGGCAUGCAGGCCUGGAU GGGG, and AAAAGCUGGGUUGAGAGGGU individually. The specific primers/sequences for amplifying miRNAs and mRNA are listed in Table 2.

TABLE 2 | Primers for reverse transcription-polymerase chain reaction.

\begin{tabular}{lll}
\hline Genes & Primers & $\begin{array}{l}\text { Accession } \\
\text { number }\end{array}$ \\
\hline hsa-miR-4498 & F: 5'-AACAATTGGGCTGGCAGGG-3' & Ml0016860 \\
hsa-miR-1226-5p & R: 5' 5'-AAGTGCAGGGTCCGAGGT-3' & Ml0006313 \\
& R: 5'-GTGCAGGGTCCGAGGT-3' & \\
hsa-mir-320c & F: 5'-ACACAAGAAAAGCTGGGTGAGA-3' & Ml0003778 \\
& R: 5'-CAGTGCAGGGTCCGAGGT-3' & \\
Ctss & F: 5'-TGGATCACCACTGGCATCTCTG-3' & NM_004079 \\
Trem2 & R: 5'-GCTCCAGGTGTGAAGCATCA-3' & \\
& F: 5'-ATGATGCGGGTCTCTACCAGTG-3' & NM_018965 \\
Ccr2 & R: 5'-GCATCCTCGAAGCTCTCAGACT-3' & \\
& F: 5'-CAGGTGACAGAGACTCTTGGGA-3' & NM_000647 \\
& R: 5'-GGCAATCCTACAGCCAAGAGCT-3' &
\end{tabular}

\section{Luciferase Reporter Assay}

The sequences of Ctss $3^{\prime}$-UTR comprising the miR-4498 binding site were synthesized and inserted into the pMIRREPORT $^{\mathrm{TM}}$ vector (Ambion) to construct a luciferase vector. Next, miRNA mimics/miR-NC (synthesized by GenePharma, Shanghai, China) and the above-mentioned luciferase vector were co-transfected into cells. The luminescence signal was detected by GloMax ${ }^{\circledR}$ 20/20 Luminometer (Promega) in $48 \mathrm{~h}$ after co-transfection in accordance with the protocol of DualGlo luciferase reporter assay (Promega, WI, United States). The values of the firefly luciferase assay were normalized to the Renilla luciferase assay value from the transfected phRL-null vector (Promega).

\section{Inducting mRNA Expression in THP1 Cells by Exosomes From Plasma}

The THP1 cells were differentiated into macrophages by culturing in 1640 medium supplemented with 25 nM PMA and $10 \%$ fetal calf serum for $48 \mathrm{~h}$, followed with a recovery period of $24 \mathrm{~h}$ in culture in the absence of PMA. To clarify the effect of exosome-derived miRNAs on THP1 cells, we detected the mRNA expressions of Ctss and Trem 2 in cells co-cultured with lipopolysaccharide (LPS), exosomes, or/and miR-4498 mimic (catalog no. 4464066, Thermo Fisher, United States)/miR-4498 inhibitor (catalog no. 4464084, Thermo Fisher, United States) in serum-free 1640 medium for $24 \mathrm{~h}$ with LPS induction $(n=3$ /group).

\section{Statistical Analysis}

The statistical analyses in the two groups were performed by a two-tailed Student's $t$-test. For multiple comparisons, the $p$-value was determined by two-way ANOVA, followed by a Bonferroni posttest. All analyses were performed with GraphPad Prism 5 software. A value of $p<0.05$ was considered to be statistically significant. Data are shown as mean \pm SD.

\section{RESULTS}

\section{Gene Expression Signature of Macrophages in the Atherosclerotic Aorta}

Recent research (6) revealed the transcription and the heterogeneity of macrophages in atherosclerotic aortas from Ldlr $^{-/}$mice fed with a high-fat diet by single-cell RNA-seq, followed with tSNE analysis. There existed five monocyterelated populations in atherosclerotic plaques (shown in Figure 1A). Importantly, these populations were also present in human advanced atherosclerosis. Analyses on differential gene expression and gene ontology enrichment revealed specific gene expression (total of 1,342 genes) patterns that distinguished these five clusters. We analyzed the data and selected the top 20 differentially expressed genes in each cluster of populations (shown in Table 3 ).

The heatmap shows the hierarchical clustering of differentially expressed mRNAs in aortas from mice fed with a fat diet 

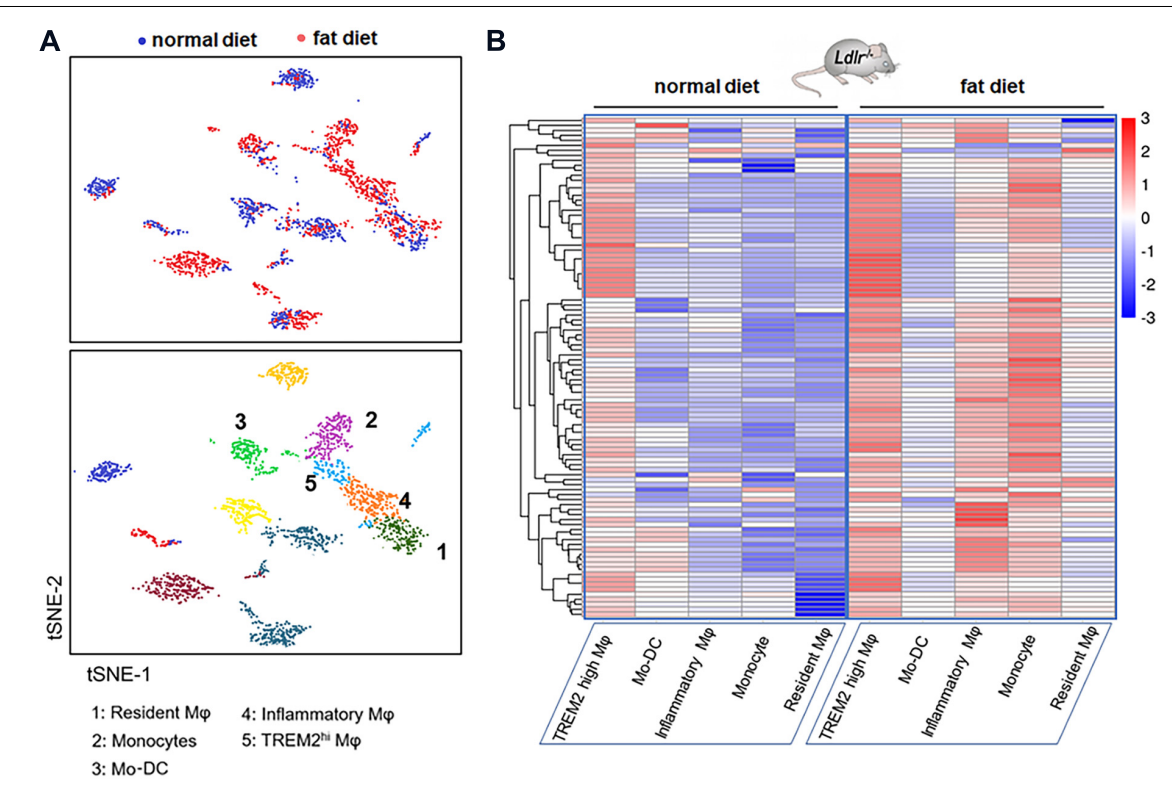

C

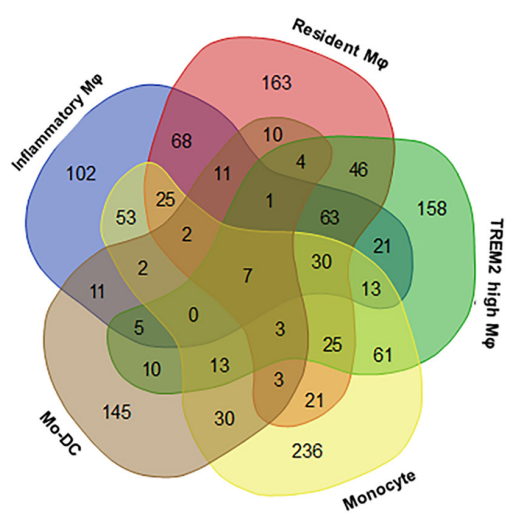

FIGURE 1 | Gene expression signature of macrophages in the atherosclerotic aorta. (A) Five monocyte-related populations in atherosclerotic aortas from Ldlr ${ }^{-/-}$ mice fed with a high-fat diet by single-cell RNA-seq, followed with tSNE analysis (data from Clement et al.). (B) Heatmap of differentially expressed genes in five clusters. Genes were ordered by hierarchical clustering. (C) Venn diagram of the gene characteristics of the five clusters.

compared with normal control in each cluster. The heatmap of genes in each $\mathrm{M} \varphi$ population vs. the four others as determined by single-cell differential expression analysis is shown in Figure 1B. The Venn diagram showed that there were seven co-expressed mRNAs (Tyrobp, Irf5, Pirb, Ctsh, Csf2ra, Spi1, and Ccdc109b) shared in five clusters, and there were $36 \mathrm{mRNAs}$ shared in at least

TABLE 3 | The top 20 differentially expressed genes in five monocyte-related populations.

\begin{tabular}{|c|c|c|c|c|}
\hline TREM2 $2^{\text {high }} \mathbf{M} \varphi$ & Resident $\mathbf{M} \varphi$ & Inflammatory $\mathbf{M} \varphi$ & Monocytes & $M \varphi-D C$ \\
\hline Trem2 & $\mathrm{C} 1 \mathrm{qb}$ & $\mathrm{C} 1 \mathrm{qb}$ & Lgals3 & Syngr2 \\
\hline $\mathrm{C} 1 \mathrm{qb}$ & C1qa & C1qa & Psap & Cd209a \\
\hline Anxa5 & Ctsc & $\mathrm{C} 1 \mathrm{qc}$ & Msrb1 & $\mathrm{H} 2-\mathrm{Aa}$ \\
\hline $\lg 1$ & C1qc & Nfkbiz & F10 & Ifi30 \\
\hline Cd9 & Lyz2 & Cd83 & Cstb & $\mathrm{H} 2-\mathrm{Ab} 1$ \\
\hline Cd63 & Sepp1 & $\mathrm{Cxcl} 2$ & Plin2 & Gm2a \\
\hline Hexb & Pf4 & Egr1 & Rnh1 & $\mathrm{Cd} 74$ \\
\hline $\mathrm{C} 1 \mathrm{qc}$ & Fcgrt & Socs3 & Tgfbi & Flt3 \\
\hline Lgals3 & Csf1r & Zfp36 & Thbs1 & $\mathrm{H} 2-\mathrm{Eb} 1$ \\
\hline Ctsb & Trf & Junb & Lilrb4a & Napsa \\
\hline Ms4a7 & Cd81 & C5ar1 & Osm & Olfm1 \\
\hline Prdx1 & Mrc1 & Csf1r & Sat1 & H2afz \\
\hline $\mathrm{Cd} 72$ & Fcrls & Nfkbia & Lst1 & H2-DMb1 \\
\hline Ctsz & Pid1 & C3ar1 & Fcer1g & H2-DMa \\
\hline Syngr1 & Serinc3 & Atf3 & Ctss & Atox 1 \\
\hline Hexa & Fcgr3 & Cd14 & Litaf & Lsp1 \\
\hline Ctss & Adgre1 & Cd81 & Ctsb & Cbfa2t3 \\
\hline Cd68 & Ehd4 & Marcksl1 & Npc2 & Plbd1 \\
\hline Ctsd & Txnip & Fcer1g & Tyrobp & $\mathrm{H} 2-\mathrm{DMb} 2$ \\
\hline C1qa & Cfh & Adgre1 & Msr1 & Rogdi \\
\hline
\end{tabular}

four clusters, such as Cst3, Ctsz, Il10rb, Cd63, Ctss, Ctsa, Lamp2, Sirpa, Apobec1, Fcer1g, Apoe, Fcgr3, Hexa, etc. (Figure 1C).

\section{Enrichment Analysis of GO and Pathway on Differentially Expressed Genes}

The GO enrichment analysis revealed the biological process targeted by these differentially expressed genes: antigen processing and presentation, immune system process, innate immune response, regulation of cell proliferation, and positive regulation of peptidyl-tyrosine phosphorylation (Figure 2A). The gene ontology molecular function includes protein binding, MHC class II protein complex binding, peptidase activity, IgG receptor binding, lipoteichoic acid-binding, and beta-N-acetyl hexosaminidase activity (Figure 2B). The gene ontology cellular components include lysosome, MHC class II protein complex, extracellular exosome, extracellular space, cell surface, membrane, and plasma membrane (Figure 2C). The pathway enrichment analysis (KEGG) revealed lysosome, phagosome, antigen processing and presentation, TNF-signal pathway, transcriptional mis-regulation in cancer, and cell adhesion molecules. These signaling pathways and target genes may play critical roles in regulating the immune response, including the function and the phenotype of $\mathrm{M} \varphi$, shown in Figure 2D.

To address whether the genes enriched in macrophage subsets in murine atherosclerotic aortas could be detected in human lesions, we performed a literature screen, which revealed that the genes enriched in inflammatory macrophages (Tnf- $\alpha$, Tnfsf9, Ccl3, Tlr2, Egr1, and Ccl2), Res-like macrophages (Cxcl4, Lyve1, Txnip, and Gas6), and TREM ${ }^{\text {hi }}$ macrophages (Trem2 and Spp1) were detected in lesional macrophages isolated from carotid 

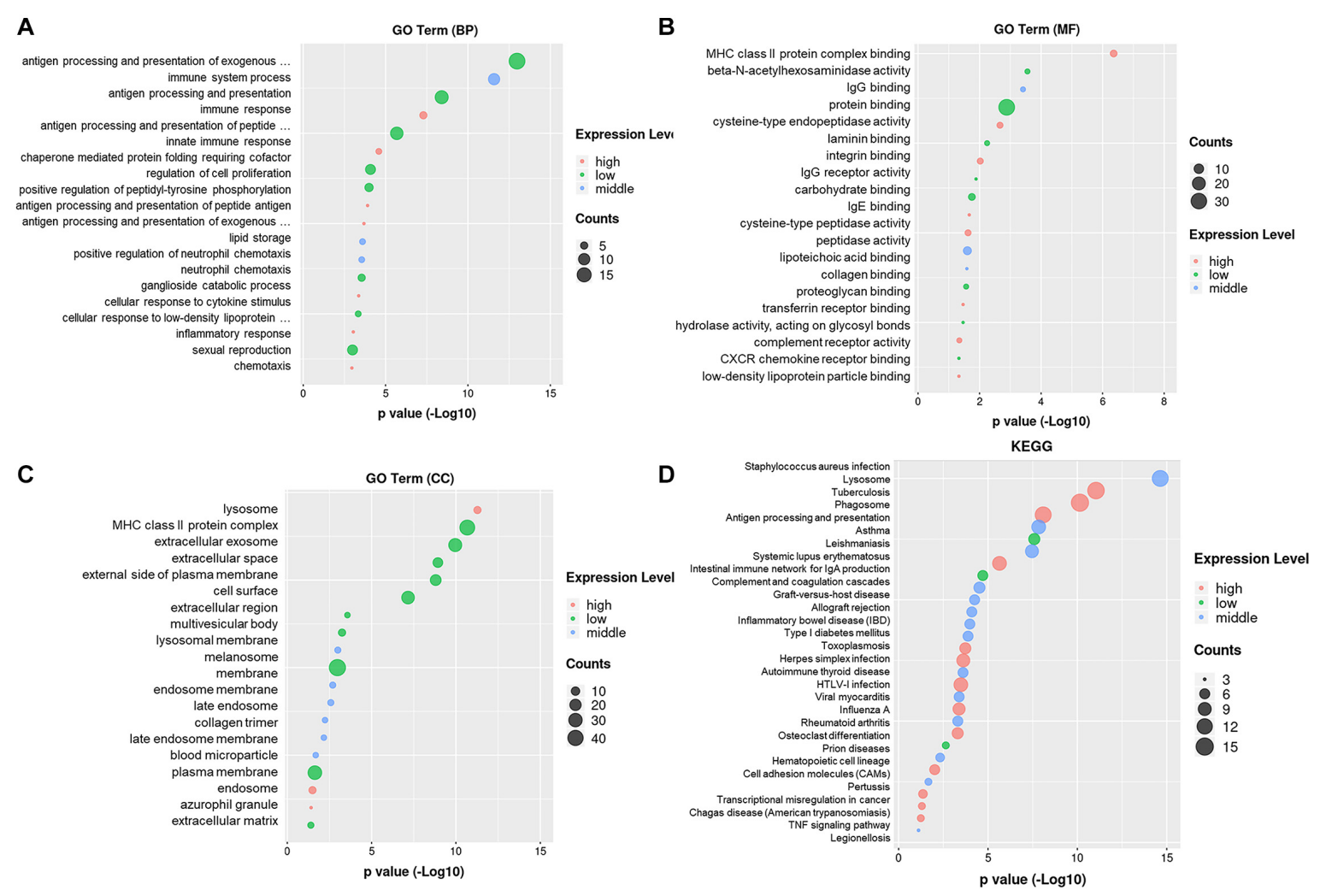

FIGURE 2 | Gene ontology enrichment and KEGG analysis on DEmRNAs. (A) Gene ontology enrichment analysis on genes relative with the biological process. (B) Gene ontology enrichment analysis on genes relative with the molecular function. (C) Gene ontology enrichment analysis on genes relative with the cellular component. (D) Pathway enrichment analysis (KEGG) on genes.

artery tissue samples from patients with high-grade carotid artery stenosis $(>70 \%)(6,11-15)$.

\section{Differentially Expressed miRNAs in Plasma Exosomes From CAD Patients and Healthy Control by miRNA Sequencing}

To explore whether the differentially expressed mRNAs were regulated by the miRNAs derived from plasma exosomes, we performed miRNA sequencing to identify differentially expressed miRNAs (DEmiRNAs) in plasma exosomes isolated from CAD patients and healthy individuals. A total of 342 known miRNAs were identified following quality control, among which 14 were relatively abundant ( $p<0.05,>1.5$-fold change) in the plasma exosomes from CAD patients vs. those from healthy individuals, including three down-regulated (hsa-miR-320c, hsa-miR-1226$5 \mathrm{p}$, and hsa-miR-4498) and 10 up-regulated miRNAs (hsamiR-452-5p, hsa-miR-196b-5p, hsa-miR-200c-3p, etc., shown in Table 4). The bar plot shows the reads count distribution of each sample by library size analysis (Figure 3A). The volcano plot showed the differentially expressed exosomal miRNAs in plasma from healthy and CAD individuals after analysis with TargetScan (fold change $>1.5$ and $p<0.05$; shown in Figure 3B). The IQR plot showed the data dispersion of each sample (Figure 3C). The scatter plot and the PCA showed the relationship and the separation of the samples between the two groups (Figures 3D,E). The heatmap showed the top differentially expressed miRNAs from plasma exosomes of healthy and CAD patients (Figure 3F).

TABLE 4 | DEmiRNAs in exosomes from coronary artery disease patients vs. control.

\begin{tabular}{llrc}
\hline & \multicolumn{1}{c}{$\boldsymbol{p}$-adj } & log2FC & $\boldsymbol{p}$-Value \\
\hline hsa-miR-452-5p & $1.60 \mathrm{E}-09$ & 23.25 & $6.36 \mathrm{E}-12$ \\
hsa-miR-196b-5p & $2.10 \mathrm{E}-09$ & 9.73 & $1.26 \mathrm{E}-11$ \\
hsa-miR-200c-3p & $3.89 \mathrm{E}-04$ & 9.66 & $7.75 \mathrm{E}-06$ \\
hsa-miR-15b-3p & $6.72 \mathrm{E}-03$ & 9.52 & $1.47 \mathrm{E}-04$ \\
hsa-miR-542-3p & $6.11 \mathrm{E}-05$ & 8.98 & $8.52 \mathrm{E}-07$ \\
hsa-miR-1304-5p & $1.94 \mathrm{E}-07$ & 8.87 & $1.93 \mathrm{E}-09$ \\
hsa-miR-29c-5p & $1.04 \mathrm{E}-04$ & 8.81 & $1.86 \mathrm{E}-06$ \\
hsa-miR-15b-5p & $8.08 \mathrm{E}-08$ & 8.57 & $6.44 \mathrm{E}-10$ \\
hsa-let-7f-1-3p & $9.21 \mathrm{E}-03$ & 7.78 & $2.39 \mathrm{E}-04$ \\
hsa-miR-433-3p & $3.45 \mathrm{E}-05$ & 7.57 & $3.02 \mathrm{E}-05$ \\
hsa-miR-221-5p & $9.00 \mathrm{E}-05$ & 7.43 & $1.43 \mathrm{E}-06$ \\
hsa-miR-320c & 0.00863 & -1.57 & 0.000206 \\
hsa-miR-1226-5p & $1.98 \mathrm{E}-07$ & -9.47 & $2.36 \mathrm{E}-09$ \\
hsa-miR-4498 & $2.12 \mathrm{E}-10$ & -24.53 & $4.23 \mathrm{E}-13$
\end{tabular}



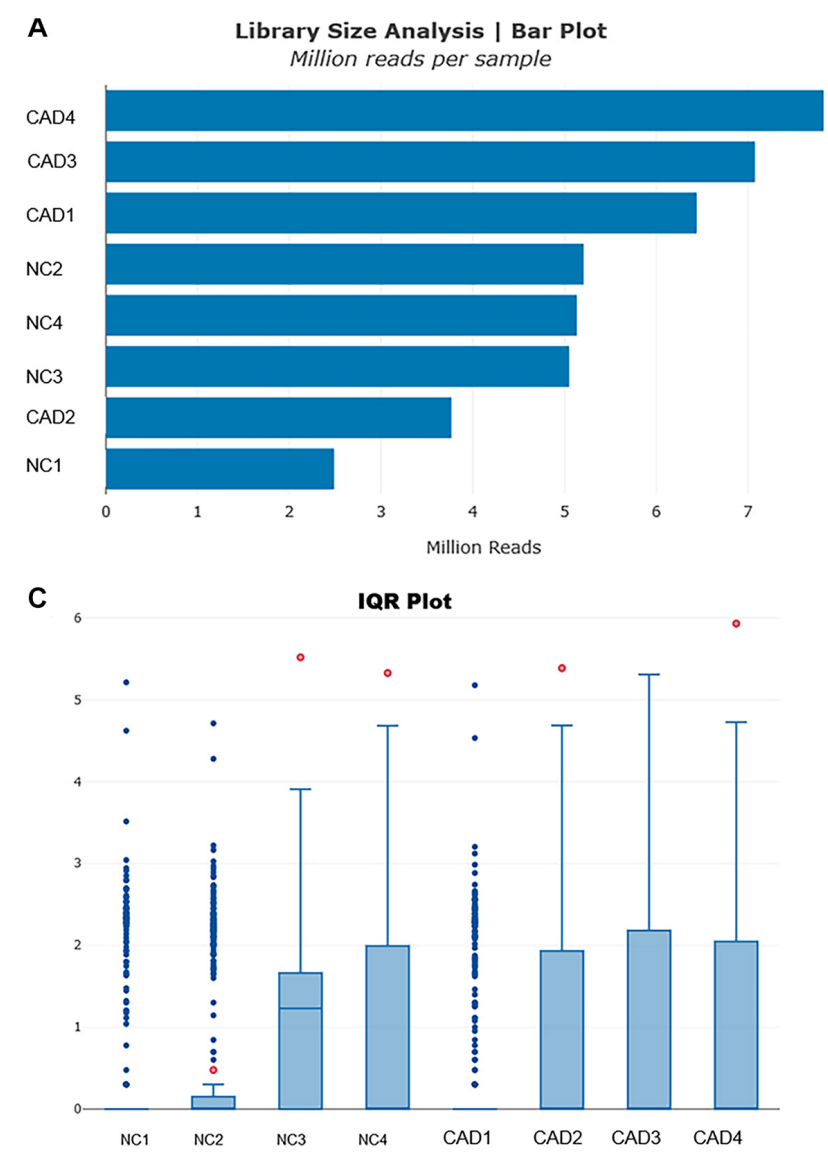

E
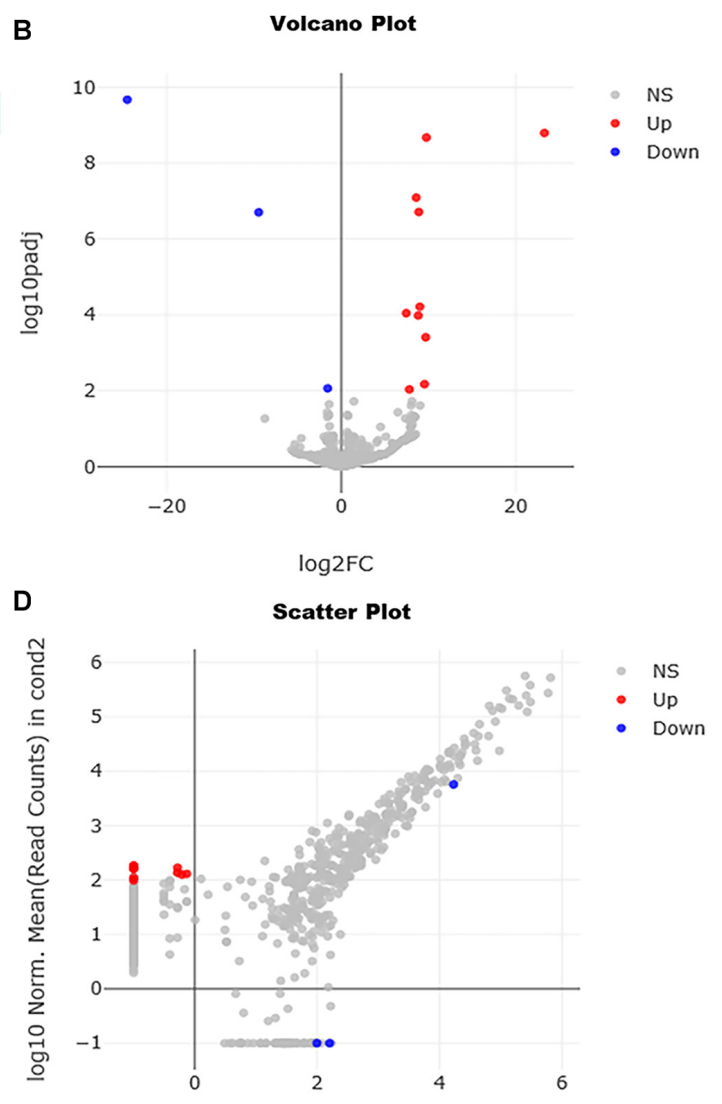

$\log 10$ Norm. Mean(Read Counts) in cond1

Heatmap Plot

$\mathbf{F}$

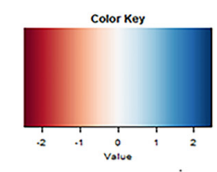

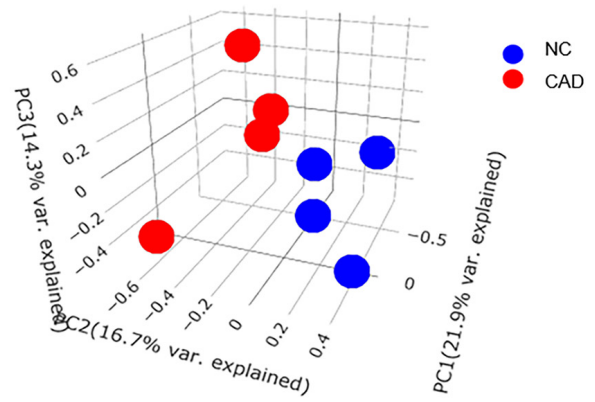

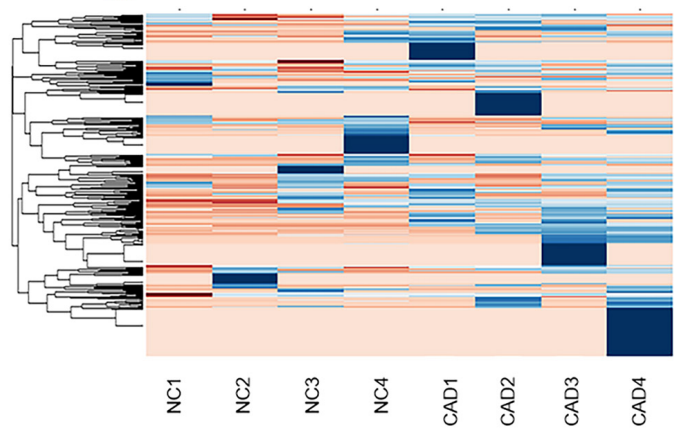

FIGURE 3 | Profiles for miRNAs of plasma exosomes from healthy and coronary artery disease (CAD) individuals. (A) Bar plot shows the reads count distribution of each sample by library size analysis. (B) Volcano plot for comparing the differentially expressed genes of exosomal miRNAs in plasma from healthy and CAD individuals after analysis with TargetScan (fold change $>1.5$ and $p<0.05$ ). (C) Inter-quartile range plot shows the data dispersion of each sample. Representative box plots of two differentially expressed miRNA that were detected in EVs from either healthy or CAD plasma are shown. (D) Scatter plot shows the relationship of read counts and the samples between the two groups. (E) Principal component analysis showing the separation of samples from healthy and CAD plasma. Each point represents a sample. (F) Heatmaps of the top differentially expressed miRNAs from plasma exosomes of healthy and CAD patients ( $n=4$ each group) were identified in hierarchical clustering. The color that represents the specific value is provided in the legend on the upper-left side of the figure.

\section{Identification of miRNA-Target mRNAs}

The miRNAs regulate the expression of specific genes by hybridization with mRNAs through MREs, thereby promoting their degradation and inhibiting their translation. To study the possible functional roles of the differentially expressed miRNAs, their potential mRNA targets were analyzed with Targetscan. 
A total of 6,379 mRNAs were predicted as the potential targets of 14 miRNAs. We obtained 5,839 target genes by deleting the duplicate and keeping the unique value (the data are not shown). Next, we identified the co-expressed mRNAs with the same expression trend both in the predicted mRNAs and the 1,342 differentially expressed genes in the above-mentioned five macrophage subset populations by manual scrutinizing. Briefly, if the expression of miRNAs was up-regulated (higher in the exosomes of CAD patients vs. healthy control), we thought that the predicted mRNAs were down-regulated in CAD patients. Then, we selected the same genes with the same expression trend (down-regulated) from 1,342 DEmRNAs in the five macrophage subset populations. A total of 38 DEmRNAs in five clusters were selected as the target genes of 14 DEmiRNAs (shown in Table 5).

\section{Construction of an Interacted Network of RNAs and Macrophage Clusters}

Based on the screening of miRNA-mRNA pairs, the coexpression networks were drawn with Cytoscape 3.7 to find out the key RNAs (Figure 4A). Each point consists of multipleweighted links to other points in the highchart to visualize the relationships among miRNAs, mRNAs, and five monocyterelated populations. The key nodes were selected by dependency wheel series $(\geq 5)$ in this network (Figure 4B). Depending on the highchart, we constructed the interacted network of key nodes, including six miRNAs (miR-433-3p, miR-320c, miR-1304-5p, miR-1226-5p, miR-452-5p, and miR-4498), 10 mRNAs (Txnip, Mrc1, Msr1, Lst1, Flt3, Cd74, Lsp1, Ms4a7, Msrb1, and Plin2), and five clusters (Figure 4C).

\section{Validation of DERNAs by Real-Time qRT-PCR}

To reveal the exact regulation on proinflammatory cytokines secreted from PBMCs by miRNAs derived from exosomes, we validated the expression of the three down-regulated miRNAs in the plasma exosomes of CAD patients in the study of RNAseq ( $n=3$ /group). The results showed that only miR-4498 by RNA-seq was significantly lower in CAD patients than in healthy control $(p<0.01)$, and there were no significant differences of miR-320c and miR-1226-5p in the two groups $(p>0.05)$ (shown in Figure 5A). To compare whether the RNA profile isolated from exosome with exoEasy Max Kit for in vitro experiments was consistent with the RNAs isolated from plasma exosomes directly with exoRNeasy Midi kits, we compared the expression of three down-regulated miRNAs by RT-PCR. The results showed the similar expression of RNA profile (Figure 5A, $n=3$ /group).

Then, we detected the mRNA expression in PBMCs from CAD patients by real-time RT-PCR to validate the previous prediction about the mRNAs which were up-regulated by the DEmiRNAs derived from the plasma of CAD patients vs. healthy control ( $n=3$ /group) in order to reveal the regulatory effect of exosomemiRNA on the expression of inflammatory cytokines in PBMCs. The results showed that the mRNA levels of Ctss, Trem2, and Ccr2 were significantly higher in CAD patients than those in healthy controls $(p<0.01)$, as shown in Figure 5B.
TABLE 5 | The DEmiRNAs/mRNAs in five monocyte-related populations.

\begin{tabular}{|c|c|c|c|c|}
\hline TREM2 high $\mathbf{M}_{\varphi}$ & C1qb & $\operatorname{miR}-15 b-5 p$ & miR-4498 & \\
\hline & Anxa5 & $m i R-1226-5 p$ & $\mathrm{miR}-4498$ & \\
\hline & $\operatorname{lgf1}$ & miR-1304-5p & $m i R-29 c-5 p$ & miR-433-3p \\
\hline & & miR-4498 & miR-320c & miR-452-5p \\
\hline & Ctsb & miR-221-5p & miR-29c-5p & $m i R-1226-5 p$ \\
\hline & & miR-4498 & & \\
\hline & Ms4a7 & $m i R-452-5 p$ & miR-4498 & \\
\hline & Syngr1 & miR-200c-3p & miR-4498 & \\
\hline & Hexa & miR-320c & & \\
\hline & Ctss & $m i R-1226-5 p$ & miR-320c & $\mathrm{miR}-4498$ \\
\hline \multirow[t]{9}{*}{$\mathrm{M} \varphi-\mathrm{DC}$} & Syngr2 & miR-4498 & $m i R-200 c-3 p$ & \\
\hline & $\mathrm{Gm} 2 \mathrm{a}$ & miR-433-3p & miR-320c & miR-4498 \\
\hline & $\mathrm{Cd} 74$ & miR-320c & miR-4498 & \\
\hline & Flt3 & $m i R-433-3 p$ & $m i R-1226-5 p$ & miR-320c \\
\hline & Olfm1 & miR-320c & miR-4498 & miR-200c-3p \\
\hline & $\mathrm{H} 2 \mathrm{afz}$ & let-7f-2-3p & $m i R-1304-5 p$ & \\
\hline & Lsp1 & miR-4498 & & \\
\hline & Plbd1 & $m i R-542-3 p$ & & \\
\hline & Rogdi & $m i R-433-3 p$ & & \\
\hline \multirow[t]{11}{*}{ Inflammatory $\mathrm{M} \varphi$} & Cd83 & $\operatorname{miR}-15 b-5 p$ & miR-29c-5p & \\
\hline & Egr1 & $m i R-1304-5 p$ & $m i R-15 b-3 p$ & \\
\hline & Socs3 & $m i R-221-5 p$ & & \\
\hline & Csf1r & miR-320c & & \\
\hline & Nfkbia & $m i R-1304-5 p$ & miR-4498 & \\
\hline & C3ar1 & $m i R-15 b-5 p$ & miR-1304-5p & \\
\hline & Cd81 & miR-433-3p & miR-4498 & miR-1226-5p \\
\hline & Marcksl1 & miR-320c & & \\
\hline & Fcer1g & $m i R-196 b-5 p$ & $m i R-433-3 p$ & \\
\hline & Msrb1 & miR-433-3p & & \\
\hline & F10 & miR-4498 & & \\
\hline \multirow[t]{7}{*}{ Monocyte } & Cstb & $m i R-1226-5 p$ & miR-4498 & \\
\hline & Plin2 & $m i R-1226-5 p$ & & \\
\hline & Sat1 & $m i R-1226-5 p$ & & \\
\hline & Lst1 & $m i R-1304-5 p$ & & \\
\hline & Msr1 & miR-433-3p & miR-320c & miR-4498 \\
\hline & Fcgrt & miR-1304-5p & & \\
\hline & Mrc1 & miR-452-5p & & \\
\hline \multirow[t]{3}{*}{ Resident $\mathrm{M} \varphi$} & Serinc3 & $m i R-1304-5 p$ & & \\
\hline & Txnip & $m i R-1304-5 p$ & miR-433-3p & miR-4498 \\
\hline & Cfh & miR-4498 & & \\
\hline
\end{tabular}

\section{Relationship Between miR-4498 Level and Coronary Artery Stenosis}

In light of the angiography findings, the CAD patients were divided into single- or multiple-vessel stenosis groups. The levels of miR-4498 were significantly lower in multiple-vessel stenosis $(n=9)$ groups than those in single-vessel stenosis $(n=6)$ groups $(p<0.01)$, and the levels in both of these two groups were downregulated compared with that in the healthy control $(n=5)$ group $(p<0.01)$, as shown in Figure 5C. In addition, we selected part of the individuals to perform a correlation analysis between the levels of miR-4498 and the percentage of coronary artery stenosis. The results showed that the levels of miR-4498 were negatively 

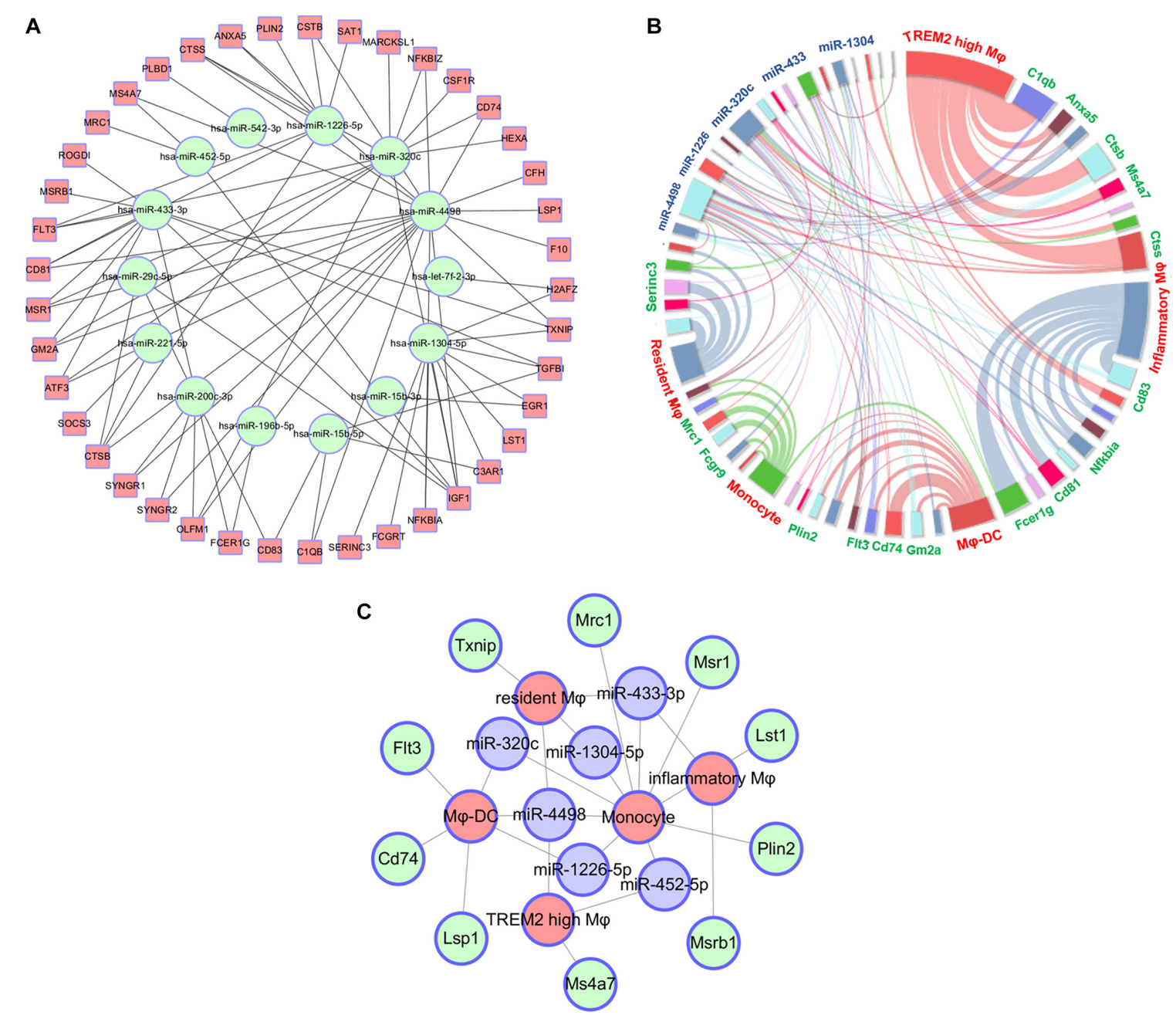

FIGURE 4 | Construct of interacted network of RNAs and monocyte-related populations. (A) The co-expression networks based on the screening of miRNA-mRNA pairs. (B) Highchart showing the relationships between miRNAs, mRNA, and five monocyte-related clusters. (C) The interacted network constructed by the key six miRNAs, 10 mRNAs, and five monocyte-related populations.

correlative to the percentage of coronary artery stenosis $(p<0.01$, $\left.R^{2}=0.9732, n=20\right)$, shown in Figure 5D.

\section{Luciferase Reporter Assay}

The results showed that the enforced expression of miR-4498 could dramatically reduce the luciferase activity of the wild-type Ctss luciferase vector $(p<0.05)$, without affecting the luciferase activity of the mutant one, but the enforced expression of miR4498 could not reduce the luciferase activity of the wild-type Ctss luciferase vector (Figure 5E, $n=3$ /group).

\section{Characterization of Plasma Exosomes}

The exosomes were characterized based on their morphology and size. The analyses by means of transmission electron microscopy confirmed the typical characterization of wholemounted exosomes (Figure 6A). The particles of the exosomes were found in the range of $40-120 \mathrm{~nm}$ by NTA, supporting a multimodal size distribution of exosomes with a peak diameter of 70-120 nm (Figure 6B), consistent with previous reports (16). The protein content of exosomes isolated from plasma was characterized by Western blotting. The plasma exosomes from each patient were positive for the exosome markers CD63 and CD9 (Figure 6C).

\section{Internalization of PKH67-Labeled Exosomes}

To address whether exosomes containing miRNAs can be internalized into THP1 cells, we first labeled the purified exosomes with the green fluorescent lipid dye PKH67 and incubated them with the cells. After incubation, green fluorescence-positive puncta were observed confluent in cultured cells (Figure 6D). The relative object integral fluorescence was measured over time with the uptake of exosomes, and the percentage of the uptake rate was calculated with the software (shown in Figures 6E,F). 

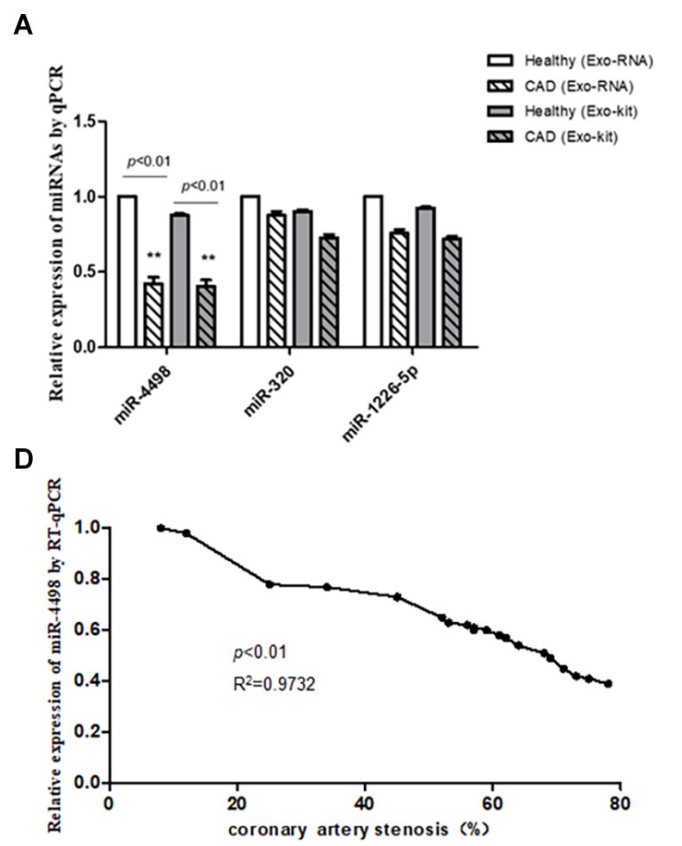

B

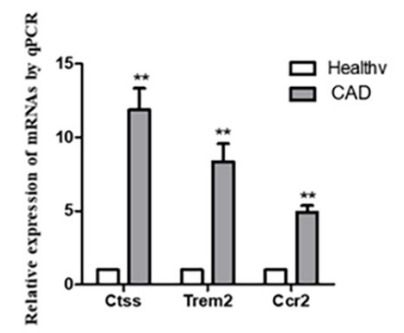

$\mathbf{E}$

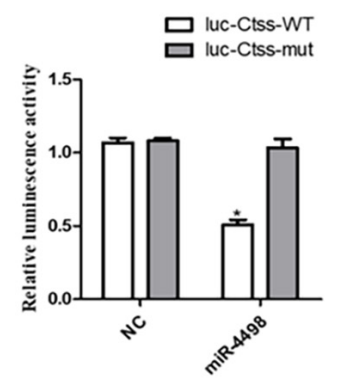

C

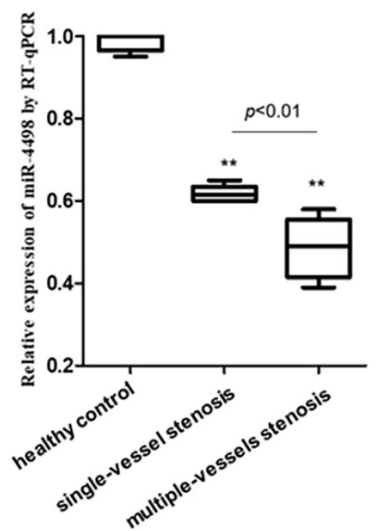

FIGURE 5 | The quantitation of RNAs to validate the predicted results. (A) The quantitation of miRNAs isolated from plasma exosomes directly with exoRNeasy Midi Kits or miRNAs in exosomes isolated with exoEasy Max Kit as detected by RT-PCR. ${ }^{* *} p<0.01$ vs. healthy control, $n=3 /$ group. (B) The quantitation of mRNAs in peripheral blood mononuclear cells was detected by RT-qPCR. ${ }^{* *} p<0.01$ vs. healthy control, $n=3 /$ group. (C) The comparison of levels of mir-4498 between the patients with single-vessel stenosis $(n=6)$ and those with multiple-vessel stenosis $(n=9)$ vs. healthy controls $(n=5)$. ${ }^{* *}<0.01$ vs. controls. (D) The correlation analysis between the levels of miR-4498 and the percentage of coronary artery stenosis in patients. $n=20$. (E) The luciferase reporter assays in THP1 cells transfected with wild-type or mutant miR-4498 luciferase vector and control. ${ }^{*} p<0.05$ vs. normal control, $n=3 /$ group.

\section{mRNA Expression in Macrophages After Uptake of Exosomes}

THP1 cells were differentiated into macrophages by PMA and cultured with lipopolysaccharide plus plasma exosomes to simulate an inflammatory microenvironment in vivo of CAD patients. Then, the cells were collected for RT-PCR assay to clarify the regulation of miR-4498 derived from exosomes. The results showed that the levels of Ctss and Trem 2 mRNA were up-regulated as expected after having been induced by LPS plus exosomes from CAD patients (CAD exo) ( $p<0.01$ vs. control), and miR-4498 mimics down-regulated the levels of both mRNAs $(p<0.01$ vs. LPS + CAD exo). While the levels of Ctss and Trem2 mRNA were down-regulated after being induced by LPS plus exosomes from healthy controls (CN exo) ( $p<0.01$ vs. LPS) and the miR-4498 inhibitor up-regulated the levels of both mRNAs $(p<0.01$ vs. LPS $+\mathrm{CN}$ exo) (shown in Figure 7$)$, we speculated that the miR-4498 derived from exosomes played a key role in regulating the mRNA levels of Ctss and Trem2.

\section{DISCUSSION}

Recent studies have shown that exosomes mediate the communication between cells and organs, which provides insights into the development of novel therapeutics for cardiovascular diseases. Researches demonstrate that the majority of miRNAs in plasma are encapsulated in exosomes (17).
Exosomes carry these important cargo molecules throughout the body, sending signals to distant tissues and coordinating the system response (18). The miRNAs that they carry provide the cells with specific features that can reveal important insights into the origin of the cells and the pathogenesis mechanism in diseases (19). In cardiovascular diseases, such as acute myocardial infarction and atherosclerosis, the miRNAs, chemokines, cytokines, growth factors, and intracellular proteins in the microenvironment play an important role, many of which are enclosed with exosomes from multiple cells, such as cardiac cells, vascular endothelial cells, and immune cells (20-23).

Atherosclerosis is characterized by plaque formation and the infiltration of inflammatory foam cell macrophages $(24,25)$. In this research, we examined monocyte-related populations in plaque by single-cell transcriptomics and exosome miRNAs from CAD patients by RNA-seq to explore the internal regulatory mechanism. It has shown that exosomes participate in the cell crosstalk and play a critical role in the pathogenesis of CAD. Therefore, a further understanding of the biological functions surrounding exosomes in the context of immunity is necessary for the development of novel therapies.

The study has shown that five atherosclerosis-associated immune cell populations could be found in atherosclerotic aortas, including CD8 + T cells, monocyte-derived DCs, monocytes, and two populations of atherosclerosis-associated macrophages (TREM2high macrophages and inflammatory macrophages) (6). Atherosclerosis-associated monocytes/macrophages showed 
A

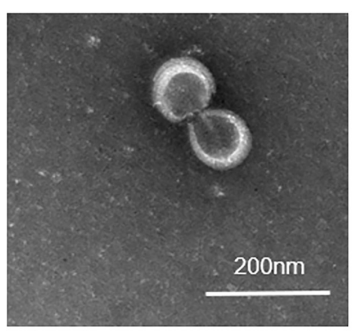

D
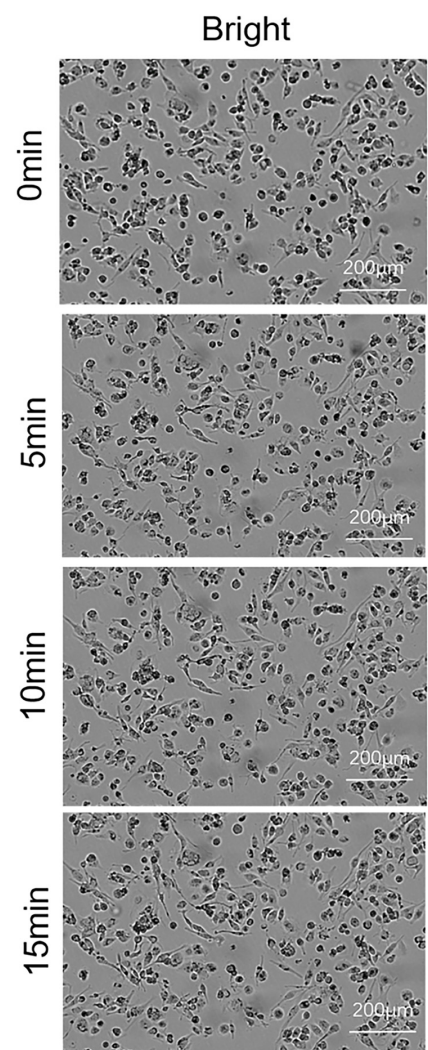

E

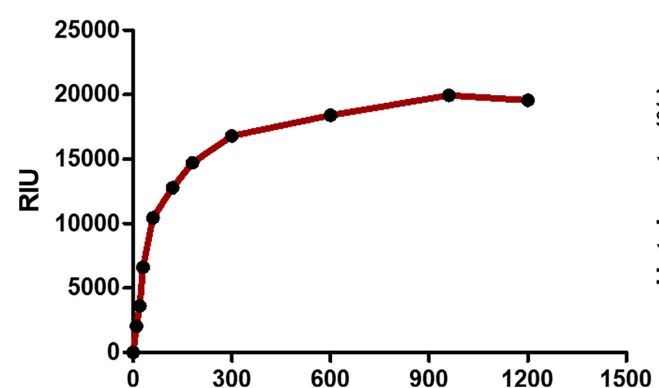

$\mathbf{F}$
B

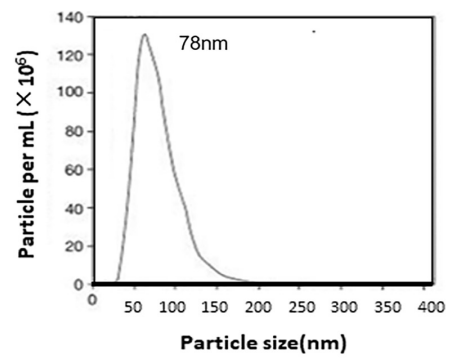

C

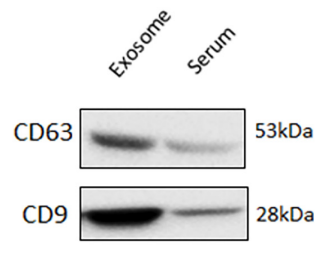

$00 \mu \mathrm{m}$

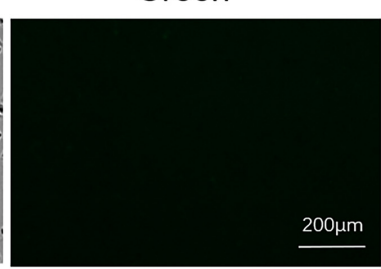

Merge
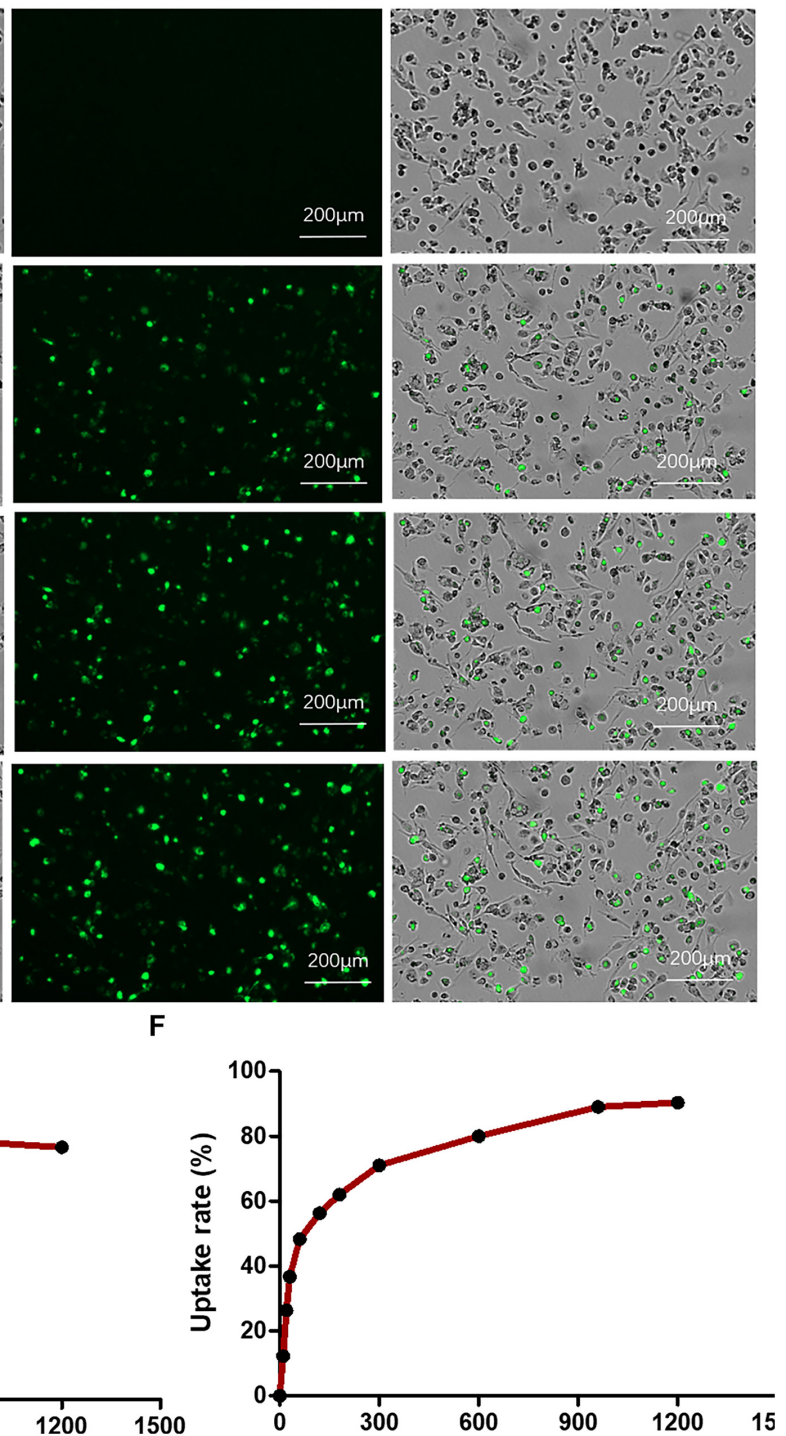

FIGURE 6 | Characterization of plasma exosomes and uptake of exosomes by macrophages. (A) Electron microscopy observation of whole-mounted exosomes purified from plasma. (B) Average overall size distribution of exosomes from plasma using nanoparticle tracking analysis. (C) The proteins from isolated exosomes were initially characterized on Western blot to assess the expression of CD63 and CD9. (D) Internalization of PKH67 (green)-labeled exosomes in THP1 cells induced by phorbol-12-myristate-13-acetate was captured by living cell imaging. Fluorescent cellular imaging was carried out using the green fluorescent protein channel for PKH67 fluorescence-labeled exosomes (green). Cell counts were detected with bright field. (E) The relative object integral fluorescence was measured over time with the uptake of exosomes. (F) The percentage of uptake rate was calculated with the software. 
A

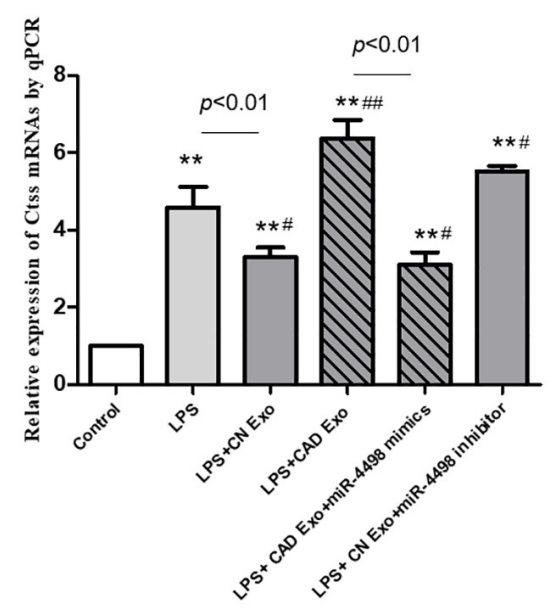

B

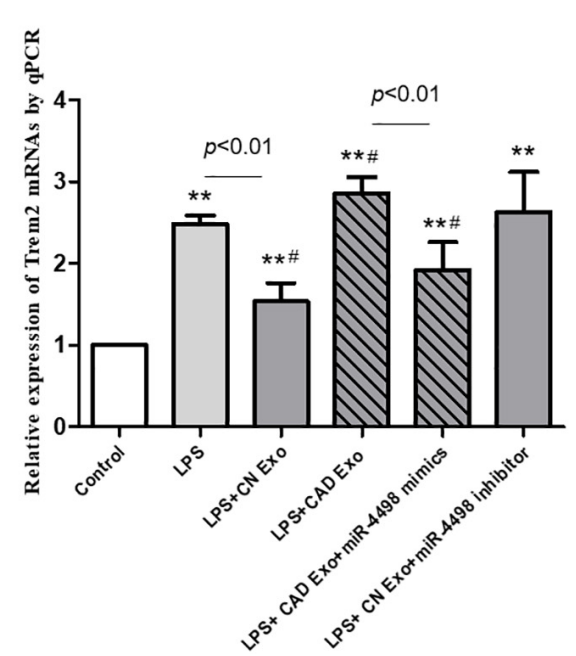

FIGURE 7 | Gene expression of macrophages induced by exosomes from the plasma. The quantitation of mRNAs of Ctss (A) and Trem2 (B) in THP1 cells was detected by RT-qPCR. ${ }^{* *} p<0.01$ and ${ }^{*} p<0.05$ vs. control, ${ }^{\# \#} p<0.01$ and ${ }^{\#} p<0.05$ vs. lipopolysaccharide; $n=3 /$ group.

high expressions of Adgre1, Cd14, Fcgr1, and Csf1r and a low expression of Ly6c2. The most significantly enriched genes in inflammatory macrophages included various proinflammatory chemokines ( $\mathrm{Cxcl} 2, \mathrm{Ccl} 3$, and Ccl4), Tlr2, and Nlrp3. TREM2 ${ }^{\text {hi }}$ macrophages displayed a unique gene signature with the expressions of Cd9, Hvcn1, and several cathepsins, except the most significantly enriched gene Trem 2 .

Ctss (cathepsin S), a member of the peptidase C1 family, is a lysosomal cysteine proteinase that participates in the degradation of antigenic proteins into peptides which are presented on MHC class II molecules $(26,27)$. When secreted from cells, this protein can remodel components of the extracellular matrix such as elastin, collagen, and fibronectin. This gene is involved in the pathology of many inflammatory and autoimmune diseases. The study has shown that the proteolytic activity of intracellular caspase 1 and extracellular Ctss in macrophages can be used as alternative biomarkers for lysosomal rupture and acute inflammation (28). Previous research demonstrated that Ctss was expressed by intimal macrophages as well as SMCs in human atherosclerotic arteries and involved in atherogenesis along with serine proteases and MMPs (29). The inhibition of Ctss could decrease the atherosclerotic lesions in $\mathrm{ApoE}^{-/-}$ mice (30).

The results confirmed our hypothesis that LPS could induce the differentiation of Trem $2^{\text {high }}$ macrophages, while miR-4498 derived from plasma exosomes could inhibit the expression of inflammatory cytokines, such as Ctss and Trem2. In exosomes from CAD patients, the levels of miR-4498 were low, and these could not inhibit the expression of inflammatory cytokines, while the high levels of miR-4498 in exosome from healthy individuals could inhibit the expression of these inflammatory cytokines effectively and reverse the differentiation of monocytes/macrophages into anti-inflammatory phenotype.

This study suggests that tissue-derived exosomal miRNA might polarize monocytes (macrophages) into pro-inflammatory phenotype and thus further accelerate macrophage infiltration and chronic atherosclerotic inflammation. Circulating exosomal miRNAs potentially contribute to regulating molecular signaling networks in cardiovascular diseases. This study provides new insight regarding the pathogenic profile of exosomes in coronary artery disease. In future studies, we will further explore the pathogenesis of atherosclerosis based on more exosomal miRNAs regulating the function of macrophages.

\section{DATA AVAILABILITY STATEMENT}

The datasets presented in this study can be found in online repositories. The names of the repository/repositories and accession number(s) can be found in the article/supplementary material.

\section{ETHICS STATEMENT}

The studies involving human participants were reviewed and approved by the institutional ethical committee of Liaoning University of Traditional Chinese Medicine. The patients/participants provided their written informed consent to participate in this study.

\section{AUTHOR CONTRIBUTIONS}

WC designed the majority of experiments. XL wrote the manuscript. XH and JW contributed to the data analysis of bioinformation. AZ helped in experimental design. DW and PC contributed to performed WB and gene assay. All authors contributed to the article and approved the submitted version. 


\section{FUNDING}

This work was supported by the National Natural Science Foundation of China (grant nos. 81874372 and 81874417), the National Key R\&D Program of China (grant no.

\section{REFERENCES}

1. Valadi H, Ekström K, Bossios A, Sjöstrand M, Lee JJ, Lötvall JO. Exosomemediated transfer of mRNAs and microRNAs is a novel mechanism of genetic exchange between cells. Nat Cell Biol. (2007) 9:654-9. doi: 10.1038/ncb1596

2. Kuwabara Y, Ono K, Horie T, Nishi H, Nagao K, Kinoshita M, et al. Increased microRNA-1 and microRNA-133a levels in plasma of patients with cardiovascular disease indicate myocardial damage. Circ Cardiovasc Genet. (2011) 4:446-54. doi: 10.1161/circgenetics.110.958975

3. Kakkar R, Lee RT. Intramyocardial fibroblast myocyte communication. Circ Res. (2010) 106:47-57. doi: 10.1161/CIRCRESAHA.109.207456

4. Eulalio A, Mano M, Dal Ferro M, Zentilin L, Sinagra G, Zacchigna S, et al. Functional screening identifies miRNAs inducing cardiac regeneration. Nature. (2012) 492:376-81. doi: 10.1038/nature11739

5. Xu Q, Seeger FH, Castillo J, Iekushi K, Boon RA, Farcas R, et al. MicroRNA-34a contributes to the impaired function of bone marrow-derived mononuclear cells from patients with cardiovascular disease. J Am Coll Cardiol. (2012) 59:2107-17. doi: 10.1016/j.jacc.2012.02.033

6. Cochain C, Vafadarnejad E, Arampatzi P, Pelisek J, Winkels H, Ley K, et al. Single-cell RNA-Seq reveals the transcriptional landscape and heterogeneity of aortic macrophages in murine atherosclerosis. Circ Res. (2018) 122:1661-74. doi: 10.1161/circresaha.117.312509

7. Friedländer MR, Chen W, Adamidi C, Maaskola J, Einspanier R, Knespel S, et al. Discovering microRNAs from deep sequencing data using miRDeep. Nat Biotechnol. (2008) 26:407-15. doi: 10.1038/nbt1394

8. Torre D, Lachmann A, Ma'ayan A. BioJupies: automated generation of interactive notebooks for RNA-Seq data analysis in the cloud. Cell Syst. (2018) 7:556-61.e3. doi: 10.1016/j.cels.2018.10.007

9. Dweep H, Gretz N. miRWalk2.0: a comprehensive atlas of microRNA-target interactions. Nature Methods. (2015) 12:697. doi: 10.1038/nmeth.3485

10. Lund ME, To J, O'Brien BA, Donnelly S. The choice of phorbol 12myristate 13-acetate differentiation protocol influences the response of THP-1 macrophages to a pro-inflammatory stimulus. J Immunol Methods. (2016) 430:64-70. doi: 10.1016/j.jim.2016.01.012

11. Maguire JJ, Jones KL, Kuc RE, Clarke MC, Bennett MR, Davenport AP. The CCR5 chemokine receptor mediates vasoconstriction and stimulates intimal hyperplasia in human vessels in vitro. Cardiovasc Res. (2014) 101:513-21. doi: $10.1093 / \mathrm{cvr} / \mathrm{cvt} 333$

12. Lutgens E, Tjwa M, de Frutos PG, Wijnands E, Beckers L, Dahlbäck B, et al. Genetic loss of Gas6 induces plaque stability in experimental atherosclerosis. J Pathol. (2008) 216:55-63. doi: 10.1002/path.2381

13. Hirota S, Imakita M, Kohri K, Ito A, Morii E, Adachi S, et al. Expression of osteopontin messenger RNA by macrophages in atherosclerotic plaques. A possible association with calcification. Am J Pathol. (1993) 143:1003-8.

14. Zhang H, Taylor WR, Joseph G, Caracciolo V, Gonzales DM, Sidell N, et al. mRNA-binding protein ZFP36 is expressed in atherosclerotic lesions and reduces inflammation in aortic endothelial cells. Arterioscler Thromb Vasc Biol. (2013) 33:1212-20. doi: 10.1161/ATVBAHA.113.301496

15. Nelken NA, Coughlin SR, Gordon D, Wilcox JN. Monocyte chemoattractant protein-1 in human atheromatous plaques. J Clin Invest. (1991) 88:1121-7. doi: 10.1172/JCI115411

16. Dragovic RA, Gardiner C, Brooks AS, Tannetta DS, Ferguson DJP, Hole P, et al. Sizing and phenotyping of cellular vesicles using nanoparticle tracking analysis. Nanomedicine. (2011) 7:780-8. doi: 10.1016/j.nano.2011.04.003

17. Hannafon BN, Trigoso YD, Calloway CL, Zhao YD, Lum DH, Welm $\mathrm{AL}$, et al. Plasma exosome microRNAs are indicative of breast
2019YFC1708701), the Key Basic Research Project of Liaoning Ministry of Education (grant no. L201701), and the Young and Middle-Aged Science and Technology Innovation Talent Support Plan of Shenyang, China (grant no. RC180069).

cancer. Breast Cancer Res BCR. (2016) 18:90. doi: 10.1186/s13058-0160753-x

18. Poe AJ, Knowlton AA. Exosomes and cardiovascular cell-cell communication. Essays Biochem. (2018) 62:193-204. doi: 10.1042/ebc20170081

19. Barile L, Cervio E, Lionetti V, Milano G, Ciullo A, Biemmi V, et al. Cardioprotection by cardiac progenitor cell-secreted exosomes: role of pregnancy-associated plasma protein-A. Cardiovasc Res. (2018) 114:992-1005. doi: $10.1093 / \mathrm{cvr} / \mathrm{cvy} 055$

20. Chistiakov DA, Orekhov AN, Bobryshev YV. Cardiac extracellular vesicles in normal and infarcted heart. Int J Mol Sci. (2016) 17:63. doi: 10.3390/ ijms 17010063

21. Eguchi S, Takefuji M, Sakaguchi T, Ishihama S, Mori Y, Tsuda S, et al. Cardiomyocytes capture stem cell-derived, anti-apoptotic microRNA-214 via clathrin-mediated endocytosis in acute myocardial infarction. J Biol Chem. (2019) 294:11665-74. doi: 10.1074/jbc.RA119.007537

22. de Couto G, Gallet R, Cambier L, Jaghatspanyan E, Makkar N, Dawkins JF, et al. Exosomal MicroRNA transfer into macrophages mediates cellular postconditioning. Circulation. (2017) 136:200-14. doi: 10.1161/ CIRCULATIONAHA.116.024590

23. Zheng B, Yin WN, Suzuki T, Zhang XH, Zhang Y, Song L, et al. Exosomemediated miR-155 transfer from smooth muscle cells to endothelial cells induces endothelial injury and promotes atherosclerosis. Mol Ther. (2017) 25:1279-94. doi: 10.1016/j.ymthe.2017.03.031

24. Rasheed A, Cummins CL. Beyond the foam cell: the role of LXRs in preventing atherogenesis. Int J Mol Sci. (2018) 19:2307. doi: 10.3390/ijms19082307

25. Bäck M, Yurdagul A Jr., Tabas I, Öörni K, Kovanen PT. Inflammation and its resolution in atherosclerosis: mediators and therapeutic opportunities. Nat Rev Cardiol. (2019) 16:389-406. doi: 10.1038/s41569-019-0169-2

26. Campden RI, Zhang Y. The role of lysosomal cysteine cathepsins in NLRP3 inflammasome activation. Arch Biochem Biophys. (2019) 670:32-42. doi: 10. 1016/j.abb.2019.02.015

27. Liu W, Spero DM. Cysteine protease cathepsin S as a key step in antigen presentation. Drug News Perspect. (2004) 17:357-63. doi: 10.1358/dnp.2004. 17.6.829027

28. Hughes CS, Colhoun LM, Bains BK, Kilgour J, Burden R, Burrows JF, et al. Extracellular cathepsin $\mathrm{S}$ and intracellular caspase 1 activation are surrogate biomarkers of particulate-induced lysosomal disruption in macrophages. Part Fibre Toxicol. (2016) 13:19. doi: 10.1186/s12989-016-0129-5

29. Sukhova GK, Zhang Y, Pan JH, Wada Y, Yamamoto T, Naito M, et al. Deficiency of cathepsin S reduces atherosclerosis in LDL receptor-deficient mice. J Clin Invest. (2003) 111:897-906. doi: 10.1172/JCI14915

30. Samokhin AO, Lythgo PA, Gauthier JY, Percival MD, Brömme D. Pharmacological inhibition of cathepsin S decreases atherosclerotic lesions in Apoe-/- mice. J Cardiovasc Pharmacol. (2010) 56:98-105. doi: 10.1097/FJC. ob013e3181e23e10

Conflict of Interest: The authors declare that the research was conducted in the absence of any commercial or financial relationships that could be construed as a potential conflict of interest.

Copyright (c) $2020 \mathrm{Li}, \mathrm{He}$, Wang, Wang, Cong, Zhu and Chen. This is an open-access article distributed under the terms of the Creative Commons Attribution License (CC BY). The use, distribution or reproduction in other forums is permitted, provided the original author(s) and the copyright owner(s) are credited and that the original publication in this journal is cited, in accordance with accepted academic practice. No use, distribution or reproduction is permitted which does not comply with these terms. 